\title{
Review suggests that cleft lip and palate patients have more caries
}

\author{
Abstracted from \\ Antonarakis GS, Palaska PK, Herzog G. \\ Caries prevalence in non-syndromic patients with cleft lip and/or palate: a meta-analysis. \\ Caries Res 2013; 47: 406-413. doi: 10.1159/000349911. Epub 2013 May 3. PubMed PMID: 23652859.
}

\section{Question: Is there a difference in caries prevalence between cleft lip and palate (CLP) and a non-CLP population?}

Data sources The Cochrane library, Medline, Embase, CINAHL and Scopus databases were searched.

Study selection Cross-sectional or longitudinal studies evaluating caries prevalence in CLP patients against a suitable matched control were included.

Data extraction and synthesis Study selection, data extraction and quality assessment were carried out independently by two reviewers. Meta-analysis was carried out using a random effects model.

Results Seven studies (total 474 patients) met the inclusion criteria. All of the studies were cross-sectional in nature, and used the decayed, missing and filled (DMF/dmf) indices as the final outcomes. Five studies involving permanent teeth suggested that CLP patients have a higher number of DMF teeth than the controls (mean difference 1.38; $p=0.003$ ). Four studies of primary teeth suggest that CLP patients have a higher number of $\mathrm{dmf}$ teeth than the controls (mean difference $1.51 ; \mathrm{p}=0.03$ ).

Conclusions Individuals with CLP, when compared with matched non-CLP controls, tend to have a higher prevalence of dental caries, as detected using the decayed, missing and filled index. This holds true both for permanent and for deciduous teeth. One must keep in mind, however, that relatively few studies were included in the present metaanalysis, and the reporting on the methods in the individual studies was not always transparent.

\section{Commentary}

This meta-analysis addresses an important oral health issue where well-designed studies are lacking: caries prevalence among individuals with cleft lip and/or palate. A meta-analysis is a statistical method of combining the results from studies, which meet established inclusion and exclusion criteria, to produce an overall estimate of effect from results included. While the meta-analysis allows for similar studies to be combined, there is, nevertheless, heterogeneity even in similar studies. Antonarakis et al. note that there was substantial heterogeneity for the DMFT and dmft scores of the seven studies included in their meta-analysis, and the authors provide an overview of the shortcomings of the available research in this area. The authors discuss one limitation of studies; that they pool all children with CLP into one group, regardless of cleft type, and pooling does not allow for precise assessment of caries prevalence (eg patients with bilateral clefting are at increased risk for caries compared to individuals with a unilateral cleft) ${ }^{1}$. Second, only three of the studies included in the meta-analysis used matched controls for sociodemographics which play a significant role in caries experience $^{2}$.

In addition, the authors have outlined the considerable variability in the studies regarding each one's rigorousness of adherence to the WHO survey method. All of the studies included in this metaanalysis each individually conclude that caries experience is higher for individuals with CLP except for the Lucas et al. [2000] study. ${ }^{3}$ These authors suggest there was no statistical difference between the CLP and control groups because children with clefting were examined more frequently by healthcare personnel than controls. Hence, future studies could 'match' controls on frequency of access to dental care. Nevertheless, Antonarakis et al. iterate the multiple challenges that face individuals with CLP (malocclusion, food impaction, orthodontic appliances, enamel hypoplasia) that are probable reasons for individuals with clefting to experience higher rates of caries. This is the first article, to my knowledge, to use the meta-analysis methodology to quantify the differences in caries prevalence among individuals with CLP and controls. While the results of this analysis allude to the challenges of epidemiological studies, they provide quantified data which indicate that individuals with CLP have higher caries prevalence than non-CLP controls, and this information can be used for health care planning.

\section{Martha Wells}

Department of Pediatric Dentistry, University of Tennessee Health Science Center, Memphis, Tennessee, USA

1. Wells M. Oral health status of children with craniofacial anomalies. Pediatr Dent 2013; 35: E79-86.

2. Council on Clinical Affairs American Academy of Pediatric Dentistry: Guideline on caries-risk assessment and management for infants, children, and adolescents. Pediatr Dent 2013; 35: E157-164.

3. Lucas VS, Gupta R, Ololade O, et al. Dental health indices and caries associated microflora in children with unilateral cleft lip and palate. Cleft Palate Craniofac J 2000; 37: $447-452$

Evidence-Based Dentistry (2014) 15, 79. doi:10.1038/sj.ebd.6401042 